\title{
Color Information Processing in Higher Brain Areas*
}

\author{
Hidehiko Komatsu $^{1,2}$ and Naokazu Goda ${ }^{1,2}$ \\ ${ }^{1}$ National Institute for Physiological Sciences, Okazaki, Japan \\ ${ }^{2}$ The Graduate University for Advanced Studies (SOKENDAI), Okazaki, Japan \\ komatsu@nips.ac.jp
}

\begin{abstract}
Significant color signal transformation occurs in the primary visual cortex and neurons tuned to various direction in the color space are generated. The resulting multi-axes color representation appears to be the basic principle of color representation throughout the visual cortex. Color signal is conveyed through the ventral stream of cortical visual pathway and finally reaches to the inferior temporal (IT) cortex. Lesion studies have shown that IT cortex plays critical role in color vision. Color discrimination is accomplished by using the activities of a large number of color selective IT neurons with various properties. Both discrimination and categorization are important aspects of our color vision, and we can switch between these two modes depending on the task demand. IT cortex receives top-down signal coding the task and this signal adaptively modulates the color selective responses in IT cortex such that neural signals useful for the ongoing task is efficiently selected.
\end{abstract}

\section{Neural Pathway for Color Vision}

Visual systems in the human and monkey brains have functional differentiation and consists of multiple parallel pathways [1]. Color information is carried by specific types of retinal cells and transmitted along specific fibers in the optic nerve [2][3]. Visual signals leaving the eye are relayed at the lateral geniculate nucleus (LGN) and then reach to the primary visual cortex (or V1) situated at the most posterior part of the cerebral cortex. LGN has multi-layered organization, and color information is coded only at specific layers. Cerebral cortex contains a number of visual areas, and these areas consist of two major streams of visual signals. Of these, color information is carried by the ventral visual stream that is thought to be involved in visual recognition of objects. Ventral visual stream starts from sub-regions in V1, include sub-regions of area V2, area V4 and finally reaches to the inferior temporal cortex (or IT cortex) (Fig. 1).

In humans, damage in the ventral cortical area around fusiform gyrus results in the loss of color sensation (achromatopsia), so this area should play a critical role in color vision. In the macaque monkey, a very good animal model of human color vision, IT cortex plays a very important role in color vision because selective damage in the IT

* This work is supported by a Japanese Grant-in-Aid for Scientific Research (B) and a grant for Scientific Research on Priority Areas from MEXT of Japan. 


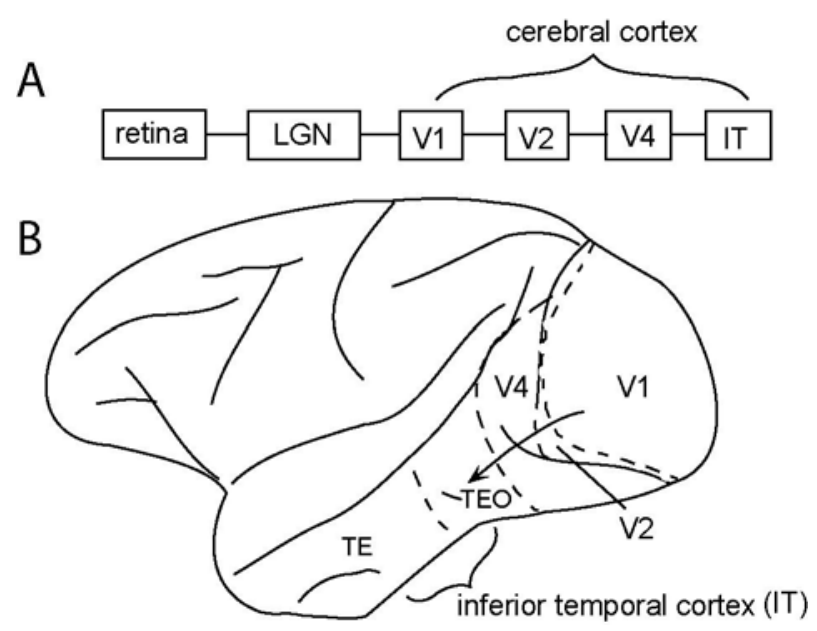

Fig. 1. Visual pathway in the monkey brain related to color vision. V1: primary visual cortex, V2: area V2, V4: area V4, IT: inferior temporal cortex. TE and TEO correspond to the anterior and posterior part of IT.

cortex results in severe deficit in color discrimination [4-6]. In this paper, we will describe our researches on how the color information is represented and transformed at different stages of the visual pathway, and how the neuron activities in the IT cortex are related to the behavior using color signals.

\section{Representation of Color Information}

Color vision originates from the comparison of signals of photoreceptors with different spectral sensitivity functions. Humans and macaque monkeys have three types of cone photoreceptors that are maximally sensitive to long (L), middle (M) and short (S) wavelengths, and they are called $\mathrm{L}$ cone, $\mathrm{M}$ cone and $\mathrm{S}$ cone, respectively. Comparison of signals from different types of cones occurs in the retinal circuit, and the resulting difference signals are sent to LGN through the optic nerve. At this stage, it has been known that color information is carried by two types of color selective neurons, namely, red-green (R/G) color opponent neuron, and blue-yellow (B/Y) color opponent neuron. The former type of cells code the difference between L-cone and M-cone signals (either L-M or M-L). On the other hand, the latter type of cells code the difference between S-cone signal and the sum of the signals from the remaining two types of cones $(\mathrm{S}-(\mathrm{L}+\mathrm{M}))$.

Different laboratories have used different color stimuli to characterize the color selectivity of neurons. In our laboratory, we have used color stimuli based on the CIExy chromaticity diagram [7][8]. To study the color selectivity of a neuron, we used a set of color stimuli that were systematically distributed on the chromaticity diagram and mapped the responses on the diagram (Fig.2). Each color stimulus had the same luminance, shape and area. Color stimuli were presented on the computer display one by one at the same position in the receptive field of the recorded neuron. We employed CIE-xy chromaticity diagram because of the general familiarity of this 

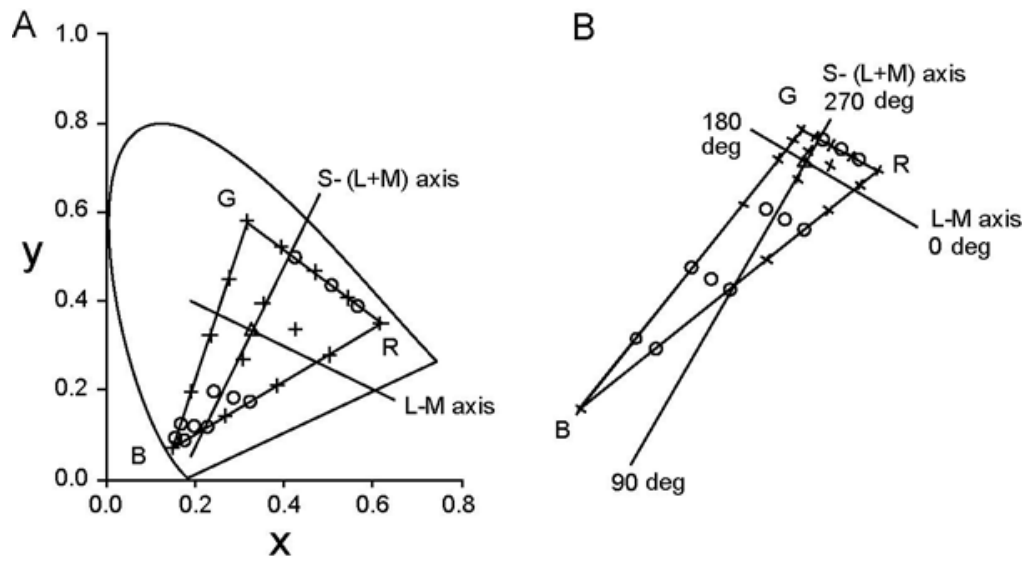

Fig. 2. Color stimuli used in our laboratory that were systematically distributed in the chromaticity diagram. A: Colors plotted on the CIE-xy chromaticity diagram. B: Colors replotted on the MacLeod-Boynton (MB) chromaticity diagram. In both $\mathrm{A}$ and $\mathrm{B},+$ indicates the chromaticity coordinates of color stimuli distributed regularly on the CIE-xy chromaticity diagram, $\bigcirc$ those of color stimuli distributed regularly on the MB chromaticity diagram, and $\Delta$ the equal-energy white point. Cardinal axes in the MB diagram [L-M and S-(L+M)] are also shown. From [8] with modification.

diagram, and because we can easily describe the color selectivity in terms of the combination of cone signals because XYZ space on which CIE-xy diagram is based and LMS space representing cone signals are connected by linear transformation. By using this method, comparison of the color selectivity of neurons in LGN and V1 was conducted [8]. Figure 3 left shows typical examples of color selectivity of LGN neurons. Response magnitude to each color stimulus is expressed as the diameter of the circle and plotted at the position in the chromaticity diagram that corresponds to the chromaticity coordinates of the color. Open circle represents excitatory response and filled circle represents inhibitory response. Cell 1 showed strong response to red colors and exhibited no response to cyan to green colors. This is an example of $\mathrm{R} / \mathrm{G}$ color opponent neuron. Cell 2 showed strong excitatory responses to blue colors and strong inhibitory responses to colors around yellow. This is an example of B/Y color opponent neurons. In these diagrams, contour lines of the equal-magnitude responses are also plotted. Like these example neurons, LGN neurons generally had straight response contours.

To examine how the cone signals are combined to generate these neural responses, response contours were re-plotted on the MacLeod-Boynton (MB) chromaticity diagram [9] by using the cone spectral sensitivities as a transformation matrix [10], and the direction in which the response magnitude most steeply changes (tuning direction) was determined. Lower half of Figure 3 left shows the tuning directions of 38 LGN neurons recorded. They were concentrated only at very limited directions in color space: two large peaks were observed at $0 \mathrm{deg}$ and $180 \mathrm{deg}$. These correspond to the difference signal between $\mathrm{L}$ and $\mathrm{M}$ cones: 0 deg corresponds to L-M signal, and $180 \mathrm{deg}$ to $\mathrm{M}-\mathrm{L}$ signal. Altogether, these peaks correspond to the R/G color opponent 

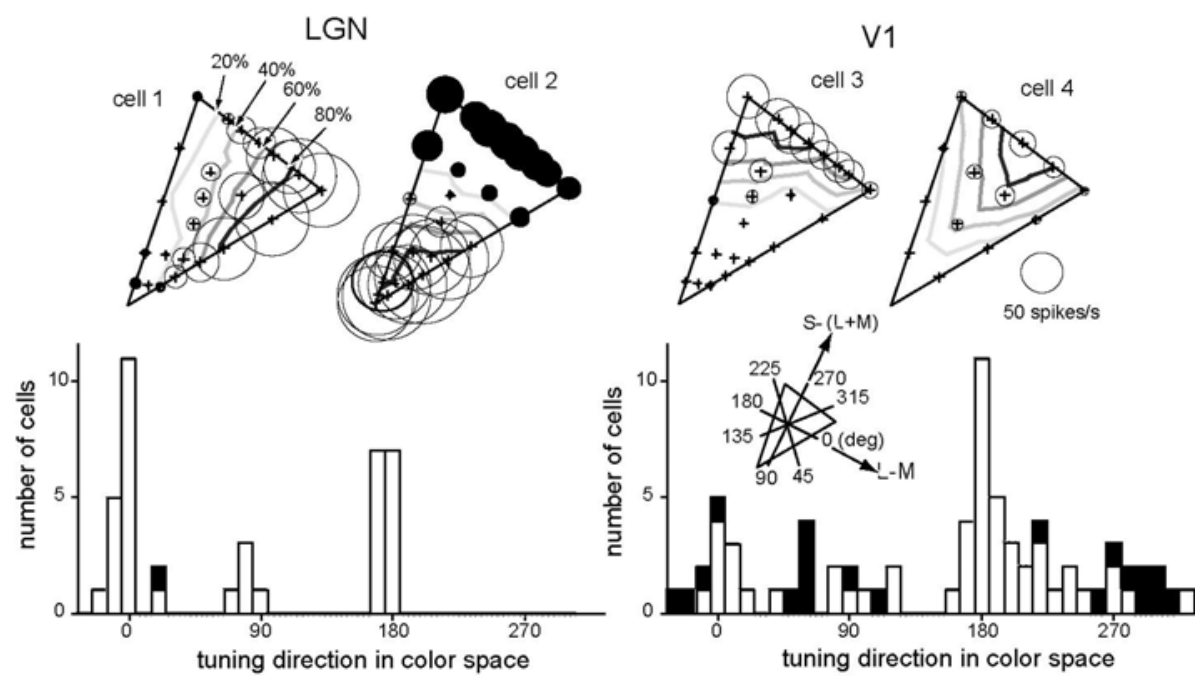

Fig. 3. Left: Color selectivity of two example LGN neurons (top) and distribution of the tuning directions of LGN neurons (bottom). Right: Color selectivity of two example V1 neurons (top) and distribution of the tuning directions of V1 neurons (bottom). See text for the detail. From [8] with modification.

neurons. There was a smaller peak at around $90 \mathrm{deg}$. This corresponds to the difference signal between $\mathrm{S}$ cone signal and the sum of $\mathrm{L}$ and $\mathrm{M}$ cone signals, namely $\mathrm{S}-(\mathrm{L}+\mathrm{M})$ signal, and represent the $\mathrm{B} / \mathrm{Y}$ color opponent neurons. We can think that, at this stage, color is decomposed along two axes that consists of MB chromaticity diagram, namely $\mathrm{L}-\mathrm{M}$ and $\mathrm{S}-(\mathrm{L}+\mathrm{M})$ axes. Different colors correspond to different weighs on each of these axes.

Color is represented in V1 in a way quite different from that in LGN. Right half of Figure 3 shows the color selectivity of two example neurons in V1. Compared with LGN neurons, there were two major differences. First, tuning directions of V1 neurons widely vary and are not restricted in certain directions as observed in LGN. The response contours of cell 3, for example, have orientation that is never observed in LGN. Figure 3 right bottom shows tuning directions of 73 V1 neurons. They are widely distributed across many directions in the color space. This indicates that different hues are represented by different neurons in V1. These results indicate that there is dramatic change in the way hue is represented between LGN and V1. Secondly, many color selective V1 neurons had clearly curved response contours (e.g. cell 4) that were unusual in LGN where neurons in principle had straight response contours. Filled parts of bar graphs in Figure 3 indicate neurons in which a model yielding curved response contours make the data fitting significantly better than any model having only straight response contours. The curved response contours enable to restrict the responses in any region in the chromaticity diagram, and can generate sharp tuning to any hue. Therefore, the neural process involved in forming the curved response contour must be closely related to the process of generating selectivity to various hues in the cerebral cortex. We can also think about the difference in color representation between LGN and V1 in the following way. The 
neural pathway connecting the eye and V1 through LGN consists of only a limited number of nerve fibers compared with the number of photoreceptors. In order to transmit visual information efficiently under such constraint, color information is encoded in a compressed form to reduce redundancy. In contrast, in the cerebral cortex, the constraint of capacity is less severe because of the large volume of the cortex, and different computational principle may dominate. It appears that visual cortex took a strategy to explicitly represent different hues with different neurons. Presumably, there is some biological advantage of representing hue independently by different set of neurons.

\section{Transformation of Color Signals in Early Visual Areas}

Difference in color selectivities of neurons between LGN and V1 indicates that significant transformation of color signal takes place in V1. Although actual neural processing for the transformation is not known, two-stages model shown in Figure 4A can explain the properties of V1 neurons quite well. At the first stage of the model, signals from $\mathrm{R} / \mathrm{G}$ color opponent neuron and $\mathrm{B} / \mathrm{Y}$ color opponent neuron are linearly summed with various combination of weights and then the resulting signal is rectified. As the result of this first stage, neurons with straight response contours tuned to various directions in the color space are formed. At the second stage, the signals from multiple cells at the first stage are linearly summed and then rectified. As the results of linear summation and rectification repeating twice, color selective neuron with curved response contours sharply tuned to specific hue are formed. Figure 4B
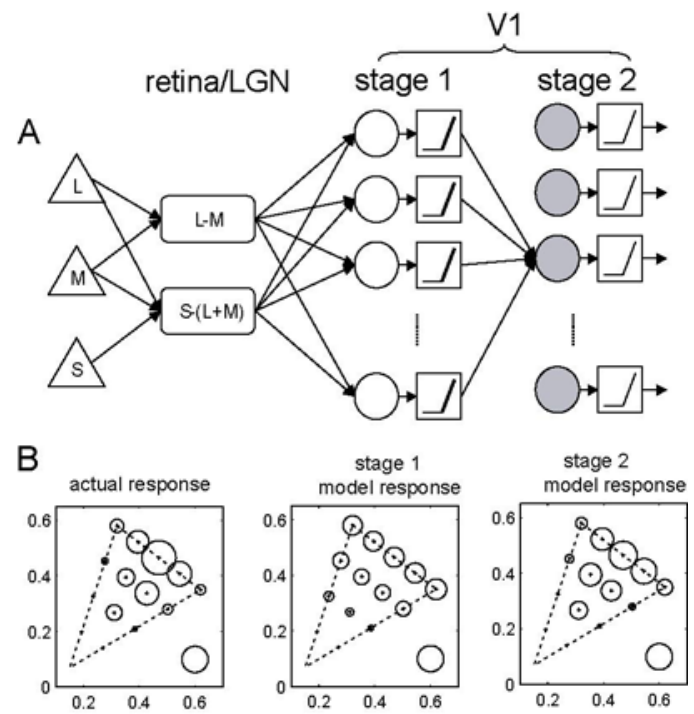

Fig. 4. A: Two-stage model to explain the transformation of color selectivity in V1. B: Responses of an example V1 neuron tuned to yellow (left), the outputs of the best model with a single stage (middle), and the outputs of the best model with double stages (right). 
illustrates the responses of an example V1 neuron tuned to yellow (real response), the outputs of the best model with a single stage, and the outputs of the best model with double stages.

\section{Relationship between Neural Responses and Behavior in the Inferior Temporal Cortex}

Color selectivity tuned to specific hue is commonly observed in each stage of the cortical visual pathway [11-15], and we believe this is the fundamental principle for color representation in the cerebral cortex. Inferior temporal cortex (IT cortex), the highest stage of the ventral stream, is thought to play a critical role in color vision because its lesion cause severe deficits in color discrimination. Color selective neurons tuned to specific hues are also found in IT cortex [7] (Fig. 5). To study how color selective IT neurons contribute to color discrimination, the quantitative relationship between color judgment in monkeys and the responses of color selective IT neurons were examined [16]. Neuronal activities and behavior recorded simultaneously while the monkeys performed a color judgment task were compared. Color discrimination threshold was computed based on the responses of each color selective IT neuron. To do this, we first computed the probability distribution function of the response magnitudes for each color, and receiver-operating-characteristic (ROC) analysis was conducted to compute the probability that an ideal observer can discriminate two different colors separated at a certain distance in the color space. Then, color discrimination threshold of individual neuron was computed based on the relationship between the color difference and the probability of the correct response. When the color discrimination thresholds based on the neuron activities and those
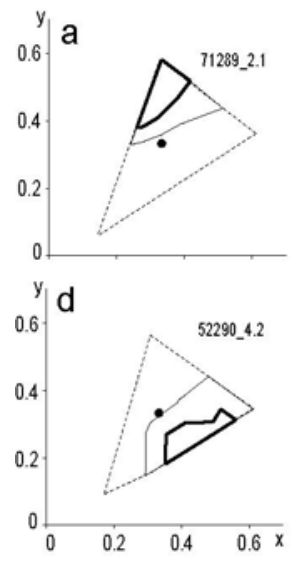
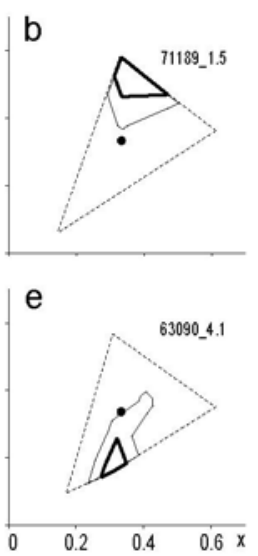
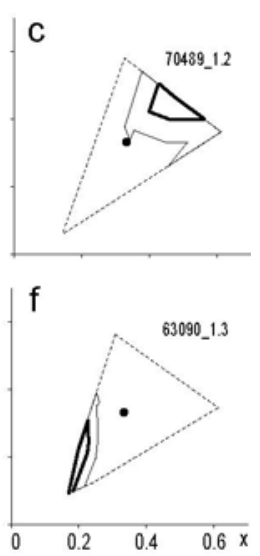

Fig. 5. Color selectivity of six examples neurons recorded from the IT cortex tuned to different hues. Color selectivity of each neuron is represented as the response contours within the region in the CIE-xy chromatcity diagram examined (broken line). In each panel, the thicker (thinner) response contour indicates the locations where the responses are $75 \%(50 \%)$ of the maximum response. From [7] with modification. 
based on the monkey's behavior were compared, neural color discrimination threshold was on average 1.5 times larger than that of the monkey, indicating that neural sensitivity tended to be somewhat lower than the behavioral sensitivity.

On the other hand, it was found that there was a strong positive correlation between neuron activity and monkey's behavior with regard to the way discrimination threshold depends on color. CIE-xy chromaticity diagram is not a uniform color space; in other words, even if two pairs of color had the same distance on the CIE-xy chromaticity diagram, their differences may not be the same perceptually [17]. As the result, discrimination threshold obtained by the monkey's behavior changes depending on the position in the chromaticity diagram. It was examined how the neural color discrimination threshold depends on the position in the chromaticity diagram and how it is related to the behavioral threshold. To do this, the chromaticity diagram was divided into 10 areas and the average discrimination thresholds in each area was computed for both neuron and behavior. Relationship between the mean neural and behavioral thresholds across different areas of the chromaticity diagram for one monkey is shown in Figure 6. There was a strong positive correlation between these two values and this clearly indicates that activities of color selective IT neurons are closely correlated with the color discrimination behavior of the monkeys. To study how individual IT neurons contribute to the monkey's color judgment, the correlation between the trial-to-trial fluctuation of the neural responses and the color judgment of the monkey was examined by the ideal-observer analysis [16]. It was found that contribution of individual neuron is relatively small and that there is no systematic relationship between the sensitivity to color difference or the sharpness of the color selectivity of the neurons and the degree to which each neuron contribute to the color judgment. These results suggest that signals from a large population of color selective neurons with various properties, rather than a small subset of neurons with especially high sensitivity, contribute to color perception and color discrimination behavior.

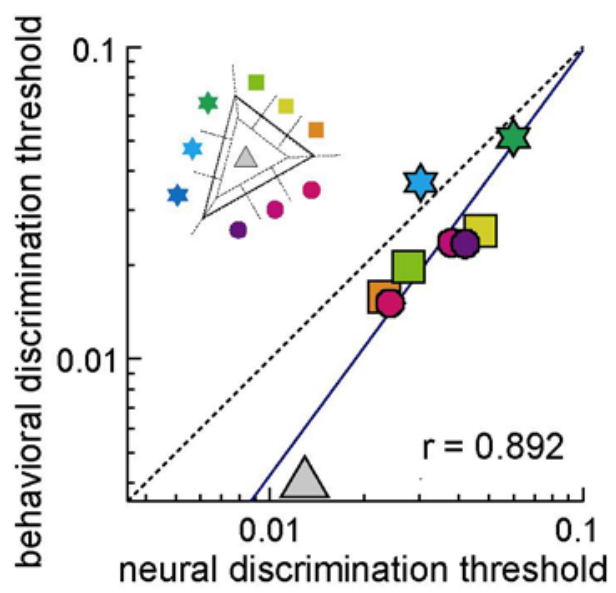

Fig. 6. Relationship between the mean neural and behavioral thresholds across different areas of the chromaticity diagram for one monkey. Each symbol corresponds to different region in the CIE-xy chromaticity diagram. See text for more detail. From [16] with modification. 


\section{Cognitive Control of Color-Related Behavior}

In our daily life, we often treat similar though perceptually distinguishable colors in the same way as a group and give the same name (e.g. green, red). Such categorical perception is an important cognitive function and it enables to efficiently manage the infinitely variable objects and events in the environment by our cognitive system with finite resource for information processing. Such categorical perception is an important aspect of our color perception. On the other hand, we make fine discrimination of similar colors in certain situations such as when we scrutinize the food or clothes at the shop. We can switch between these two functions, namely categorization and fine discrimination, depending on the situation or the task demands. It is believed that the prefrontal cortex plays central role in such cognitive control of visual behaviors [18]. However, it is not well understood how the neural responses in the visual cortical areas are affected by the top-down signal from the prefrontal cortex coding the current situation and how they are modulated depending on the task demands.

In order to understand how the cognitive control of color-related behavior using color stimuli involves neurons in the visual cortex, the activities of the color selective IT neurons of monkeys trained to perform a color categorization task and a fine discrimination task were analyzed [19]. While a single IT neuron was recorded, above two tasks were switched and it was examined how the neural responses change. In each task, 11 color stimuli that were separated in a constant interval between red and green on the CIE-xy chromaticity diagram were used. In the categorization task, one of the 11 colors was presented as the sample, and the monkey judged whether the sample was red or green. In the discrimination task, one of the 11 colors was presented as the sample and that was followed by two similar colors (choice colors), and the monkey had to select one of these choice colors that was the same as the sample. In this latter task, the monkey had to discriminate colors even though both were within the same color category (red or green). A majority of neurons $(64 \%)$ exhibited significant change in their responses to the sample color depending on the ongoing task. Responses of four example neurons to the 11 sample colors are shown in Figure 7. A large majority of these neurons (77\%) showed stronger responses in the categorization task, and the responses during passive viewing were similar to those during the categorization task. These results suggest that the default of the cognitive control is categorical judgment, and that IT neurons are in general more active in this condition. It was also shown that, as the results of response change, neural signals differentiating red category vs green category is enhanced during the categorization task and suppressed during the discrimination task. Thus, the top-down signal adaptively modulates the gain of the neural responses in IT cortex such that neural signals useful for the ongoing task is efficiently selected. Interestingly, the color selectivity of the neuron itself does not change despite the change in the response amplitude, so these neurons can transmit precise color information regardless of the task. This is in marked contrast from the prefrontal cortex where neurons exhibits selectivity corresponding to the categorical judgment [20]. These results suggest that cognitive control of visual behavior by the top-down signal from the prefrontal cortex coding the current situation or ongoing task demand involves the adaptive modulation of neuron activities in the visual cortex that carry precise sensory information. 

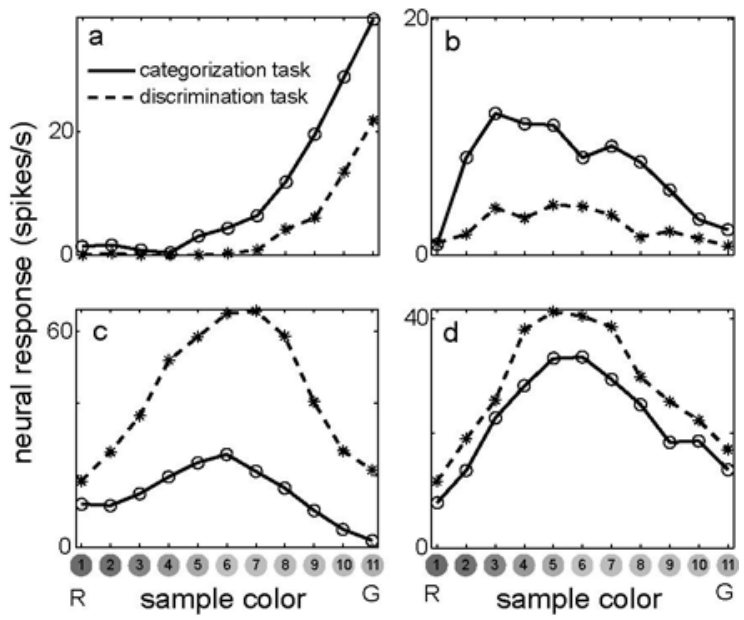

Fig. 7. Responses of four example IT neurons that were modulated by the ongoing task. Responses to the 11 sample colors recorded during the categorization task (solid line) and those during the discrimination task (broken line) are indicated. Cells in a and b exhibited stronger responses during the categorization task, and those in $\mathrm{c}$ and $\mathrm{d}$ during the discrimination task. See text for more detail. From [19] with modification.

Figure 8 schematically illustrates how the color signals originating from the three types of cones are transformed in the visual system, and how the cognitive control of behavior using color stimuli are executed by the top-down signal from the prefrontal

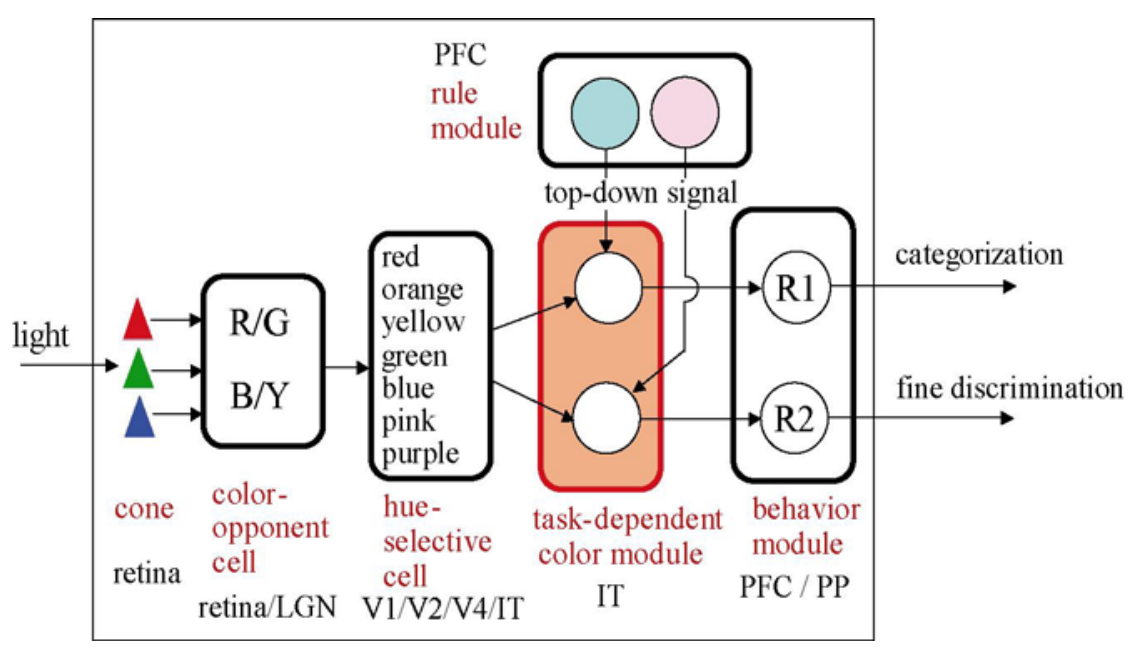

Fig. 8. Schematic illustration of the color signal transformation and cognitive control of color signals in the brain. IT: inferior temporal cortex, PFC: prefrontal cortex, PP: posterior parietal cortex. See text for more detail. 
cortex to the IT cortex. This schema is a hypothetical one based on incomplete experimental data and it lacks several important aspects of color processing such as the spatial processing of color signals. Nevertheless, color information can be basically described in the three dimensional space because it stems from the activities of three types of cones, and because of this, understanding of the neural processing of color vision is probably the most advanced field across the entire visual neuroscience. In this schema, we aimed to provide what we think is the essence of the neural processing of color itself or hue, and hope this will be a useful schema to guide further development of the research in color vision.

\section{References}

1. Maunsell, J.H., Newsome, W.T.: Visual processing in monkey extrastriate cortex. Annu. Rev. Neurosci. 10, 363-401 (1987)

2. Komatsu, H.: Mechanisms of central color vision. Curr. Opin. Neurobiol. 8, 503-508 (1998)

3. Solomon, S.G., Lennie, P.: The machinery of colour vision. Nat. Rev. Neurosci. 8, 276286 (2007)

4. Heywood, C.A., Gaffan, D., Cowey, A.: Cerebral achromatopsia in monkeys. Eur. J. Neurosci. 7, 1064-1073 (1995)

5. Buckley, M.J., Gaffan, D., Murray, E.A.: Functional double dissociation between two inferior temporal cortical areas: perirhinal cortex versus middle temporal gyrus. J. Neurophysiol. 77, 587-598 (1997)

6. Huxlin, K.R., Saunders, R.C., Marchionini, D., Pham, H.A., Merigan, W.H.: Perceptual deficits after lesions of inferotemporal cortex in macaques. Cereb. Cortex 10, 671-683 (2000)

7. Komatsu, H., Ideura, Y., Kaji, S., Yamane, S.: Color selectivity of neurons in the inferior temporal cortex of the awake macaque monkey. J. Neurosci. 12, 408-424 (1992)

8. Hanazawa, A., Komatsu, H., Murakami, I.: Neural selectivity for hue and saturation of colour in the primary visual cortex of the monkey. Eur. J. Neurosci. 12, 1753-1763 (2000)

9. MacLeod, D.I., Boynton, R.M.: Chromaticity diagram showing cone excitation by stimuli of equal luminance. J. Opt. Soc. Am. 69, 1183-1186 (1979)

10. Smith, V.C., Pokorny, J.: Spectral sensitivity of the foveal cone photopigments between 400 and $500 \mathrm{~nm}$. Vision Res. 15, 161-171 (1975)

11. Lennie, P., Krauskopf, J., Sclar, G.: Chromatic mechanisms in striate cortex of macaque. J. Neurosci. 10, 649-669 (1990)

12. Wachtler, T., Sejnowski, T.J., Albright, T.D.: Representation of color stimuli in awake macaque primary visual cortex. Neuron 37, 681-691 (2003)

13. Kiper, D.C., Fenstemaker, S.B., Gegenfurtner, K.R.: Chromatic properties of neurons in macaque area V2. Vis. Neurosci. 14, 1061-1072 (1997)

14. Zeki, S.: The representation of colours in the cerebral cortex. Nature 284, 412-418 (1980)

15. Conway, B.R., Moeller, S., Tsao, D.Y.: Specialized color modules in macaque extrastriate cortex. Neuron 56, 560-573 (2007)

16. Matsumora, T., Koida, K., Komatsu, H.: Relationship between color discrimination and neural responses in the inferior temporal cortex of the monkey. J. Neurophysiol. 100, 3361-3374 (2008) 
17. MacAdam, D.L.: Visual sensitivities to color differences in daylight. J. Opt. Soc. Am. 32, 247-274 (1942)

18. Miller, E.K., Cohen, J.D.: An integrative theory of prefrontal cortex function. Annu. Rev. Neurosci. 24, 167-202 (2001)

19. Koida, K., Komatsu, H.: Effects of task demands on the responses of color-selective neurons in the inferior temporal cortex. Nat. Neurosci. 10, 108-116 (2007)

20. Freedman, D.J., Riesenhuber, M., Poggio, T., Miller, E.K.: A comparison of primate prefrontal and inferior temporal cortices during visual categorization. J. Neurosci. 23, 5235-5246 (2003) 